\title{
Combination of Local Ablative Therapy and Continuation of Immune Checkpoint Inhibitor (ICI) Therapy Provides Durable Treatment Response Past Oligometastatic Progression in NSCLC: A Case Report
}

\author{
Cassia R. Griswold $^{a} \quad K^{2}$ atie Kerrigan ${ }^{b} \quad$ Shiven B. Patel ${ }^{b}$ \\ aDepartment of Pharmacy, Mayo Clinic, Phoenix, AZ, USA; bDivision of Oncology, \\ Department of Internal Medicine, Huntsman Cancer Institute, University of Utah, \\ Salt Lake City, UT, USA
}

\section{Keywords}

Oligometastatic disease - Local ablative therapy · Immunotherapy · Adenosquamous NSCLC . Elderly patient

\begin{abstract}
Adenosquamous carcinoma is a rare type of non-small cell lung cancer associated with advanced disease and poor prognosis. There is limited data for the management of mixed histology disease in elderly or frail patients. A 79-year-old woman with no smoking history presented with a right upper lobe lung mass on chest $x$-ray. Biopsy of the mass demonstrated an EGFR-amplified, PD-L1 positive adenosquamous lung cancer. The mass was surgically resected but the patient was not a candidate for adjuvant chemotherapy. The patient later developed a metastatic paraspinal lesion that was successfully managed with SBRT. Approximately six months later, the patient developed adrenal metastases and pembrolizumab was initiated. After three cycles of systemic therapy, she developed subcutaneous lesions in her back and chest wall, which were managed with palliative resection. Scans demonstrate stable disease and continued responsiveness to pembrolizumab over one year from the most recent local ablative therapy. This case illustrates the potential role of local ablative therapy for oligometastatic progression, as it may confer significant benefit in elderly patients or those with a more indo-
\end{abstract}


lent disease course. Additionally, we have demonstrated that continuing immunotherapy past progression is reasonable in patients with no viable alternate therapy options, as delayed responses may occur.

\section{Introduction}

Adenosquamous carcinoma is a rare histological variant of non-small cell lung cancer, accounting for approximately $0.4-2 \%$ of all primary lung carcinomas [1]. Adenosquamous lung cancers are more likely to present in advanced stages of disease and are associated with a poorer prognosis than other histologic types [2]. Given the poor outcomes with conventional chemotherapy, molecular testing to identify potentially targetable mutations is recommended by national guidelines for tumors with mixed adenosquamous histology [3].

Non-small cell lung cancer (NSCLC) has several well-established oncogenic driver mutations (EGFR, ALK, ROS1, BRAF, KRAS, MET, RET, HER2, and NTRK) and biomarkers (PD-L1) that provide actionable targets for drug therapy $[3,4]$. Immune checkpoint inhibitors (ICI) targeting PD-1 and PD-L1 have rapidly become the standard of care in the front-line setting either in combination with chemotherapy or as monotherapy for most NSCLC patients [3]. While the favorable side effect profile of ICIs make them a preferred therapy for many patients, particularly those unable to tolerate other systemic chemotherapies, the efficacy of immune checkpoint inhibitors relies on the activation of the immune system; thus, there are concerns with a potentially delayed time to response in patients with new or symptomatic metastases [5]. Consequently, the use of localized treatment modalities in combination with ICIs for oligometastatic progression in advanced NSCLC patients may provide a double-pronged approach to provide both short-term symptom relief and a potential long-term PFS and OS benefit [6].

While many patients with advanced NSCLC will progress in a widespread fashion, a minority of patients may experience a more indolent course with progression in only a limited number of metastatic sites. Thus, this paradigm of oligometastatic progression may present an opportunity for physicians to improve patients' outcomes by providing potentially curative local ablative or surgical therapies [7, 8].

Here, we present a case of NSCLC with mixed adenosquamous histology, demonstrating a delayed response to an ICI, post oligometastatic progression. We highlight that the treatment of oligometastatic disease with local ablative or surgical therapies combined with the continuation of concurrent systemic immunotherapy may be an effective treatment strategy in patients with poor performance status who lack alternative therapy options.

\section{Case Presentation}

The patient is a 79-year-old never-smoker female who presented in May 2017 after a chest X-ray demonstrated an opacity in the right upper lobe. CT chest confirmed the presence of a $2.9 \times 2.4 \times 2.2 \mathrm{~cm}$ spiculated lesion. A CT-guided biopsy of the lesion revealed a mixed TTF1 positive, napsin positive, adeno-p40 positive squamous cell carcinoma. It was estimated that two-thirds of the tumor was composed of adenocarcinoma, and one-third of squamous cell carcinoma, based on pathologic review. PDL1 22C3 by IHC staining was notable for a TPS of $90-100 \%$ in the squamous component and $1-10 \%$ in the adenocarcinoma component. Next-generation sequencing (NGS) of the biopsied tissue lacked a targetable driver mutation. 
In June 2017, a staging PET scan demonstrated hypermetabolic paratracheal, subcarinal, and hilar lymph nodes and a brain MRI was negative for intracranial metastasis, consistent with clinical stage IIIA disease (cT1bN2M0, AJCC 7th edition). In August 2017, the patient underwent video-assisted thoracoscopic upper lobectomy (VATS) with mediastinal lymph node dissection, and her final pathologic staging was consistent with stage IIB (pT2bpN1M0 AJCC 7th edition) SCC.

Due to her poor performance status post-surgical resection, standard of care adjuvant chemotherapy was held and surveillance was initiated. In November 2017, a new left paraspinal mass was detected, and FNA was consistent with metastasis of known adenosquamous carcinoma. PDL1 22C3 by IHC staining was notable for a TPS of 31-40\%, the histologic appearance was primarily squamous, and NGS revealed no targetable mutations. Given her continued poor performance status and oligometastatic progression, palliative chemotherapy was deferred and she underwent ablative SBRT to her left paraspinal mass with good disease control.

Approximately 6 months later, she developed a new right adrenal metastasis consistent with definitive disease progression. At the time, additional local ablative therapy and palliative systemic therapy were discussed, and the patient elected to try systemic therapy for disease control. Given her frailty and ECOG PS 2, pembrolizumab monotherapy (which was offlabel at the time and later FDA approved in April 2019) was recommended based on data extrapolated from KEYNOTE-042.

Prior to her third cycle of pembrolizumab, she demonstrated disease progression in four sites with new subcutaneous chest wall and back lesions, an enlarging subpleural nodule, and a new RML central lucency. Palliative resection of the chest wall lesions was pursued given significant pain at this site, and the pathology was consistent with metastatic adenocarcinoma. NGS detected myriad new genomic alterations, including KRAS G12V, of which none were targetable.

Pembrolizumab was continued in hopes of seeing a delayed effect from treatment, given the patient was adverse to, and a poor candidate for, second line cytotoxic chemotherapy. CT scans continue to show responsiveness to palliative pembrolizumab monotherapy more than 15 months after initiation and 1 year beyond initial disease progression.

\section{Discussion}

Adenosquamous lung cancers are rare, behave aggressively, and have poorer prognosis than lung tumors of non-mixed histology [2]. Molecular profiling and PD-L1 IHC are an important component of the diagnostic workup in adenosquamous NSCLC, and should be routinely incorporated into clinical practice, as molecular findings may guide treatment selection [3]. Despite her never smoking status, our patient was without a targetable driver mutation, but PD-L1 expression was high. Due to a combination of performance status and patient preference, we opted for surveillance, rather than standard adjuvant chemotherapy, after surgical resection. Shortly after surgical resection, however, she developed oligometastatic progression and local ablative therapy with SBRT was utilized with good disease control for $>6$ months.

Many case reports and early phase clinical trials have demonstrated potential benefits of local ablative therapy for at least synchronous oligometastatic progression in advanced NSCLC, but we have very limited phase III data to guide this treatment approach [7]. In the phase II SABR-COMET study, local ablative therapy with SABR to 5 or fewer sites of oligo- 
metastatic disease lead to both a statistically significant PFS and OS benefit for included patients, although only $18 \%$ of the patients in the experimental SABR arm had primary metastatic NSCLC [8]. The median age of patients included in this study was 67 and consequently it is not clear how this data can be extrapolated to older, frailer patients.

Recent clinical trial data has demonstrated significant improvements in PFS and OS outcomes in advanced NSCLC patients treated with ICIs in the front-line and subsequent settings as compared to standard of care single agent chemotherapy $[9,10]$. This has led to the widespread adoption of ICIs in the thoracic oncology clinic, despite challenges in tumor response assessment and appropriate patient selection criteria for ICIs. Notably, ECOG PS 2 patients were excluded from the majority of these clinical trials and the application of these ICI clinical trial outcomes to said patients is unclear $[11,12]$. We know that for patients without targetable mutations, single agent chemotherapy remains the standard of care after progression of disease on front line therapy. Unfortunately, prospective clinical trials evaluating chemotherapy typically enroll limited numbers of elderly patients, and the toxicities and survival outcomes demonstrated in these trials may not be may not generalizable to this patient population $[13,14]$. As such, local ablative therapies with SBRT may be a potential treatment strategy in cases of oligometastatic progression in this patient population, as they can potentially spare our frail patients from chemotherapy toxicities.

After post-surgical oligometastatic progression, our patient was treated with SBRT with a prolonged 6 month period of disease control and time off therapy. At the time of her second limited progression, a single agent ICI was initiated, although SBRT could have been considered again in this setting. Despite continued oligometastatic progression in 4 new sites approximately 9 weeks after ICI initiation, her ICI was continued, given her frailty and hesitancy to start chemotherapy. While clinical trials have shown similar response times between pembrolizumab and non-immunotherapy treatment arms, post-market data have demonstrated delayed time to response in certain subsets of patients $[5,9,10,15]$. As such, we have demonstrated that in this case it is reasonable to continue ICI past initial radiographically documented progression if patient's clinical status or personal preference precludes cytotoxic chemotherapy.

This case highlights that patients can experience delayed benefit from ICI, and that given its good tolerability, it should be considered in elderly patients without other therapy options past progression. Additionally, we demonstrated that local ablative therapies for oligometastatic progression can be effective and safe in elderly, frail patients, and give them prolonged time off of therapy with potential improved quality of life. As always, clinicians should weigh the risks and benefits of continuing therapy past progression, and have an individualized approach in the clinic, as treatment past hyperprogression would not be advisable.

\section{Acknowledgement}

We would like to acknowledge our radiation and surgical oncology colleagues for their support in the management of our thoracic oncology patients. We are also incredibly grateful to our patient for their willingness to share the details of their treatment in order to advance clinical practice. 


\section{Statement of Ethics}

The patient discussed in this report consented to publication of this case, including the details of her treatment history. All academic and clinical activities referenced in this manuscript comply with available guidance for human subjects research and are congruent with the World Medical Association Declaration of Helsinki ethical mandates.

\section{Disclosure Statement}

The authors have no conflicts of interest to declare.

\section{Funding Sources} therein.

No funding was required for the completion of this manuscript or the clinical efforts

\section{Author Contributions}

S.P. proposed the topic of the case study. C.G. and K.K. wrote the manuscript in consultation with S.P. All authors provided critical feedback and helped shaped the content of the manuscript.

\section{References}

1 Travis WD, Brambilla E, Burke AP, Marx A, Nicholson AG. WHO Classification of Tumours of the Lung, Pleura, Thymus and Heart. Lyon: International Agency for Research on Cancer; 2015.

2 Naunheim KS, Taylor JR, Skosey C, Hoffman PC, Ferguson MK, Golomb HM, et al. Adenosquamous lung carcinoma: clinical characteristics, treatment, and prognosis. Ann Thorac Surg. 1987 Nov;44(5):462-6.

3 Ettinger DS, Wood DE, Aggarwal C, Aisner DL, Akerley W, Bauman JR, et al. NCCN Clinical Practice Guidelines in Oncology: Non-Small Cell Lung Cancer. Version 3.2019. Available at NCCN.org [accessed Sept 10, 2019].

4 Villalobos P, Wistuba II. Lung cancer biomarkers. Hematol Oncol Clin North Am. 2017 Feb;31(1):13-29.

5 Borcoman E, Nandikolla A, Long G, Goel S, Le Tourneau C. Patterns of response and progression to immunotherapy. In: 2018 ASCO educational book. Volume 38. Chicago (IL): ASCO; 2018. pp. 169-78.

6 Bauml JM, Mick R, Ciunci C, Aggarwal C, Davis C, Evans T, et al. Pembrolizumab after completion of locally ablative therapy for oligometastatic non-small cell lung cancer: a phase 2 trial. JAMA Oncol. 2019 Jul;5(9):1283-90.

7 Juan O, Popat S. Ablative therapy for oligometastatic non-small cell lung cancer. Clin Lung Cancer. 2017 Nov;18(6):595-606.

8 Palma DA, Olson R, Harrow S, Gaede S, Louie AV, Haasbeek C, et al. Stereotactic ablative radiotherapy versus standard of care palliative treatment in patients with oligometastatic cancers (SABR-COMET): a randomised, phase 2, open-label trial. Lancet. 2019 May;393(10185):2051-8.

9 Herbst RS, Baas P, Kim DW, Felip E, Pérez-Gracia JL, Han JY, et al. Pembrolizumab versus docetaxel for previously treated, PD-L1-positive, advanced non-small-cell lung cancer (KEYNOTE-010): a randomised controlled trial. Lancet. 2016 Apr;387(10027):1540-50.

10 Mok TS, Wu YL, Kudaba I, Kowalski DM, Cho BC, Turna HZ, et al.; KEYNOTE-042 Investigators. Pembrolizumab versus chemotherapy for previously untreated, PD-L1-expressing, locally advanced or metastatic non-small-cell lung cancer (KEYNOTE-042): a randomised, open-label, controlled, phase 3 trial. Lancet. 2019 May;393(10183):1819-30.

11 Carmichael JA, Wing-San Mak D, O’Brien M. A Review of Recent Advances in the Treatment of Elderly and Poor Performance NSCLC. Cancers (Basel). 2018 Jul;10(7):E236. 
12 Passaro A, Spitaleri G, Gyawali B, de Marinis F. Immunotherapy in Non-Small-Cell Lung Cancer Patients With Performance Status 2: Clinical Decision Making With Scant Evidence. J Clin Oncol. 2019 Aug;37(22):1863-7.

13 Maione P, Rossi A, Sacco PC, Bareschino MA, Schettino C, Ferrara ML, et al. Treating advanced non-small cell lung cancer in the elderly. Ther Adv Med Oncol. 2010 Jul;2(4):251-60.

14 Gridelli C, Shepherd FA. Chemotherapy for elderly patients with non-small cell lung cancer: a review of the evidence. Chest. 2005 Aug;128(2):947-57.

15 Kurra V, Sullivan RJ, Gainor JF, Hodi FS, Gandhi L, Sadow CA, et al. Pseudoprogression in cancer immunotherapy: rates, time course and patient outcomes. J Clin Oncol. 2016;34(15_suppl):6580. 